\title{
Estimando a importância de características do ambiente construído para estimular bairros caminháveis usando Best-Worst Scaling
}

\author{
Ana Margarita Larrañaga ${ }^{1}$, Helena Beatriz Bettella Cybis ${ }^{2}$, Julián Arellana ${ }^{3}$, Luis Ignacio Rizzi ${ }^{4}$ e Orlando \\ Strambi ${ }^{5}$
}

\begin{abstract}
Resumo: O objetivo do estudo é determinar a importância dos atributos do ambiente construído para promover bairros caminháveis usando a técnica Best-Worst Scaling. Essa técnica permite identificar separadamente o impacto dos atributos e dos níveis dos atributos considerados no estudo, algo que não é possível com outros métodos de escolha tradicionais. Outra contribuição é a aplicação dessa técnica a um experimento elaborado com desenhos eficientes e com dois jogos de perguntas, estimando modelos mais precisos e diminuindo a carga cognitiva dos entrevistados. Modelos logit multinomial foram estimados para as respostas best, worst e best-worst combinadas, com dados coletados em Porto Alegre. O agrupamento das respostas foi testado através do teste da razão de verossimilhança. As importâncias dos atributos decorrentes dos modelos foram calculadas. Os resultados mostram que o julgamento e escolha dos melhores e piores atributos são realizados por processos cognitivos diferentes e a utilização de respostas best ou worst depende do foco da pesquisa.
\end{abstract}

Palavras-chave: caminhabilidade, ambiente construído, modelos de escolha discreta, best-worst.

\begin{abstract}
The purpose of this study is to determine the relative importance of the built environment attributes to promote walkable neighborhoods using best-worst scaling. This technique allows separate identification of attribute weights and attribute-level partworths, something not possible with other traditional experimental choice methods. Another contribution is the application of this technique to an experimental design structured using Efficient design, and with two sets of questions, estimating more accurate models and lowering the cognitive load of respondents. Multinomial logit models were estimated for the best, worst and best-worst pooled responses, collected in Porto Alegre. The response gathering was tested by the likelihood ratio test. The importance of the attributes resulting from the models was computed. The results show that judgment and choice for the best and worst attributes measured a different cognitive process and the use of best or worst responses depends on the focus of the research.
\end{abstract}

Keywords: walkability, built environment, discrete choice models, best-worst.

\section{INTRODUÇÃO}

Todas as viagens requerem um deslocamento a pé, seja diretamente até um destino ou até outro modo de transporte. Ruas, calçadas, parques, praças e outros lugares públicos, junto com a qualidade do ambiente urbano, têm um papel importante no estímulo ao deslocamento a pé, tornando alguns lugares mais convidativos e caminháveis do que outros.

Pesquisas realizadas (por exemplo, Baran et al., 2008; Cao et al., 2006, Cervero e Duncan, 2003; Ewing e Cervero, 2010; Frank e Engelke, 2001; Larranaga et al 2014b; Lee e Moudon, 2006) sugerem que pessoas que vivem em bairros orientados para pedestres são incentivadas a dirigir menos e caminhar mais. Bairros orientados para pedestres são caracterizados por uso de solo misto, alta co-

\footnotetext{
${ }^{1}$ Ana Margarita Larrañaga, Departamento de Engenharia de Transportes, UFRGS. (analarra@producao.ufrgs.br)

2 Helena Beatriz Bettella Cybis, Departamento de Engenharia, UFRGS

(helenabc@producao.ufrgs.br)

${ }^{3}$ Julián Arellana, Departamento de Engenharia Civil e Ambiental,

UNINORTE. (jarellana@uninorte.edu.co)

${ }^{4}$ Luis Ignacio Rizzi, Departamento de Engenharia de Transportes e

Logística, UC. (lir@ing.puc.cl)

${ }^{5}$ Orlando Strambi, Departamento de Engenharia de Transportes, Poli-

USP. (ostrambi@usp.br)
}

Manuscrito recebido em 08/03/2016 e aprovado para publicação em 22/07/2016

Este artigo é parte de TRANSPORTES v. 24, n. 2, 2016. ISSN: 2237-1346 (online). DOI:10.4237/transportes.v24i2.1091 nectividade viária e alta densidade populacional. Essas pesquisas mostram que o ambiente construído pode estimular ou desestimular o deslocamento a pé: uso de solo, sistemas de transporte e projeto urbano se unem para criar um ambiente urbano que influencia a decisão de caminhar dos indivíduos.

Diversas metodologias e índices têm surgido para descrever o ambiente para caminhada (Bradshaw 1993; Allan, 2001; Ray e Bracke, 2002; Dannenberg 2004; Krambeck, 2006; Walkscore, 2010). Estes indicadores, chamados de índices de caminhabilidade, têm sido bem sucedidos para descrever o ambiente. Entretanto, não são capazes de representar adequadamente o modo como os indivíduos percebem cada uma das características do ambiente. A importância de cada característica provavelmente seja diferente e, portanto, o impacto de medidas aplicadas para a melhoria dessas características também seja.

Este trabalho analisa a avaliação subjetiva dos residentes de Porto Alegre das características do bairro de residência, para estimular a realização de viagens utilitárias a pé (viagens com motivo/destino específico, por exemplo: trabalho, estudo, compras, etc). O objetivo do estudo é determinar a importância dos atributos do ambiente construído para promover bairros caminháveis, usando a técnica de Best-Worst Scaling. A técnica adotada analisa as escolhas dos indivíduos no contexto da teoria da utilidade aleatória e representa um método de elicitação de escolha que não tinha sido previamente aplicado na área de planejamento urbano, mas sim em outras áreas. A principal vantagem dessa abordagem é que permite a identificação separada do im- 
pacto dos atributos e impacto dos níveis dos atributos considerados na pesquisa, algo que não é possível com qualquer outro método de análise conjunta ou método de escolha tradicional. Desta forma, é possível determinar a importância dos atributos, independente dos níveis utilizados. Outro aspecto relevante a ser mencionado, corresponde à aplicação da técnica Best-Worst a um experimento elaborado com desenhos eficientes e com dois jogos de perguntas. O desenho eficiente utilizado, em lugar dos desenhos ortogonais realizados tradicionalmente, permitiu estimar modelos mais precisos (erros padrão menores). Os dois jogos considerados no experimento possibilitaram a inclusão de oito atributos, quantidade maior do que a usual neste tipo de estudo, ainda assim diminuindo a carga cognitiva dos entrevistados.

Assim, esta pesquisa permite melhor compreensão e conhecimento da importância relativa de várias características do ambiente construído. A importância relativa de cada uma permitirá identificar as características mais relevantes e com isto determinar o impacto de possíveis intervenções no ambiente urbano sobre o comportamento de pedestres. O restante do trabalho está organizado da seguinte forma. A seção 2 descreve a metodologia adotada; seção 3 discute os dados coletados, seção 4 apresenta os resultados da estimação dos modelos e discussão e seção 5 conclusões e sugestões.

\section{MÉTODO}

A abordagem proposta baseia-se na utilização da técnica Best-Worst Scaling (B/W) para analisar a importância relativa das características do bairro. A técnica tem como base os princípios da teoria da utilidade aleatória e envolve a escolha dos melhores (best) e piores (worst) atributos/níveis de atributos (os extremos mais e menos atrativos) dentro de um conjunto de situações de escolha apresentadas a cada indivíduo (Louviere e Swait, 1997).

\subsection{Best-Worst Scaling}

B/W foi introduzido pela primeira vez por Jordan Louviere em uma sessão especial de Técnicas de Pesquisa Avançada em 1988 (1988 American Marketing Association's Advanced Research Techniques Forum, ART Forum) (Burke et al, 2013) e seguido por um trabalho realizado por Louviere e Woodworth (1990). Desde então, o uso desta técnica por parte de acadêmicos tem aumentado significativamente, com aplicações em diversas áreas. Burke et al (2013) sintetizam algumas dessas aplicações: (i) marketing (por exemplo, Auger, DeVinney e Louviere, 2007; Jaeger et al, 2008; Cohen, 2009); (ii) logística de negócios (Coltman et al, 2011); (iii) economia da saúde (por exemplo, Flynn et al, 2007; Lancsar et al, 2007; Burge et al, 2011); (iv) educação (Burke et al, 2013) e (v) psicologia social (por exemplo, Lee et al 2007), para citar algumas áreas.

Recentemente, artigos começaram discutir diferentes tipos de B/W, denominados Objeto (caso 1), Perfil ou Nível de atributos (caso 2) e Multi Perfil (caso 3) (Flynn, 2010). No caso 1, objetos de estudo (que pode ser um atributo ou perfil de escolha) são apresentados ao indivíduo e este deve escolher o melhor e o pior objeto. No caso 2, perfis de escolha são apresentados ao indivíduo, um de cada vez, e este deve escolher o melhor e pior atributo dentro de cada perfil, não entre perfis. Os entrevistados escolhem o melhor e pior atributo com base nos níveis de cada atributo. Em cada perfil de escolha é apresentado unicamente um nível de cada atributo; o nível vai variando ao mudar o perfil (situação de escolha). No caso 3, os atributos são agrupados em um produto ou serviço, e vários perfis de escolha são apresentados ao indivíduo. Os entrevistados devem escolher entre perfis, o melhor e pior perfil, o que é uma extensão dos experimentos de escolha discreta tradicionais (Flynn, 2010; Adamsen e Whitty, 2013).

No presente estudo é utilizado o caso Perfil (caso 2). A escolha do método (B/W caso 2) foi devido a que neste caso é possível diferenciar entre a importância dos atributos e a importância dos níveis dos atributos. Experimentos tradicionais confundem os impactos dos atributos com os da escala utilizada para medi-los na pesquisa. Assim, a pouca (ou grande) significância estatística de parâmetros estimados em um modelo pode ser resultante de: (i) pouco (ou grande) impacto verdadeiro do atributo, (ii) limitada (ou grande) variação entre os níveis utilizados para medir esse atributo, ou (iii) ambos (Louviere e Swait, 1997). B/W supera este problema, permitindo a identificação separada dos pesos dos atributos e dos valores dos níveis dos atributos, em um contexto de análise de escolha discreta. Esta diferenciação é possível porque as escolhas B/W são realizadas dentro de um perfil de escolha, as alternativas são atributos (especificados com um determinado nível), em vez de escolha entre perfis. As utilidades estimadas são em relação a um único nível de atributo em vez de em relação a uma alternativa inteira. Os níveis dos atributos são calculados em relação a um nível base, colocando os níveis de atributos numa escala comum. A estimação de todos os níveis em uma escala comum permite a B/W calcular utilidades médias (em uma escala comum) que medem os impactos médios de atributos (Lancsar et al, 2007).

Além disso, estudos anteriores relativos à tomada de decisão sugerem que os indivíduos respondem de forma mais consistente a estímulos extremos (por exemplo, muito bom ou muito ruim) do que entre estes dois extremos (Louviere e Swait, 1997). Dados de B/W podem ser analisados por abordagens analíticas diferentes, dependendo do tipo de B/W. Modelos probabilísticos são geralmente usados para modelar as escolhas best e/ou worst para os casos Perfil (caso 2) e Multi Perfil (caso 3). Marley e Louviere (2005) desenvolveram resultados teóricos para diversos modelos probabilísticos.

No presente estudo, modelos de utilidade aleatória foram usados para modelar as respostas best, worst e escolhas agrupadas best-worst. Analisar as escolhas no contexto de utilidade aleatória implica que a posição real do nível $l$ do atributo $m$ pode ser dividida em dois componentes, um determinístico (utilidade sistemática $V_{\mathrm{ml}}$ ) e outro aleatório $\varepsilon_{m l}$ (Louviere e Swait, 1997) (Equação 1):

$$
U_{m l}^{B}=V_{m l}^{B}+\varepsilon_{m l}^{B}(1)
$$

Onde o sobrescrito B denota respostas "best". Da mesma forma, nas respostas "worst", a posição correspondente é determinada pela seguinte expressão (Equação 2):

$$
U_{m l}^{w}=V_{m l}^{w}+\varepsilon_{m l}^{w}(2)
$$

Os componentes aleatórios $\varepsilon_{m l}^{B}$ e $\varepsilon_{m l}^{W}$ são independentes e identicamente distribuídos Gumbel, com fatores de escala $\lambda^{\mathrm{B}}$ e $\lambda^{\mathrm{W}}$, em todos os atributos e níveis (Ben-Akiva e 
Lerman, 1985). Cada factor de escala está relacionado com a variância do seu componente aleatório.

Segundo Louviere e Swait (1997), admitindo que: (i) a utilidade sistemática não é afetada pela escolha dos atributos como melhores ou piores (ou seja, a utilidade sistemática do atributo é a mesma tanto se o indivíduo escolhe o melhor quanto o pior atributo): $V_{m l}^{B}=-V_{m l}^{W}=V_{m l}$; (ii) a variabilidade do termo aleatório é diferente para as respostas best e worst : $\lambda=\lambda^{W} / \lambda^{B}$

Logo, os dados das escolhas best e worst podem ser agrupados e a probabilidade de escolha de cada atributo (com seu nível) como o melhor e o pior é dado pelas seguintes expressões para modelos MNL (Equação 3) (outros modelos de utilidade aleatória podem ser usados):

$$
P_{m l}^{B}=\frac{\exp \left(V_{m l}\right)}{\sum \exp \left(V_{i j}\right)} \mathrm{e}_{P_{m l}^{W}}^{W}=\frac{\exp \left(-\lambda V_{m l}\right)}{\sum \exp \left(-\lambda V_{i j}\right)} \text { (3) }
$$

A suposição realizada em (i) e (ii) significa que os indivíduos valorizam os melhores e piores atributos da mesma forma, assim, eles tem o mesmo valor de utilidade mas multiplicada por uma constante positiva (razão de variância da escala) (Louviere e Swait, 1997). A constante é necessária para combinar dados provenientes de fontes diferentes. A suposição realizada deve ser testada para os dados coletados, e assim determinar se a hipótese é verdadeira ou não. No presente trabalho foi utilizado o teste desenvolvido por Swait e Louviere (1993) (seção 2.2).

\subsection{Modelagem}

Para testar se os entrevistados avaliam da mesma forma as melhores e piores características do bairro que estimulam a caminhada, foi adotado o procedimento de estimação proposto por Swait e Louviere (1993). Inicialmente, modelos logit multinomiais (MNL) foram estimados separadamente para as respostas best e worst, permitindo a cada um ter seus próprios parâmetros (ou seja, interceptos e coeficientes) e variabilidade de erro, usando um atributo como base. O atributo considerado como base foi o número de estabelecimentos comerciais ( $N^{o}$ comércios), por ser o atributo comum aos dois jogos do experimento. Os níveis de atributos foram codificados usando effects code (codificação de variáveis categóricas usando unicamente 1, 0 e -1). Devido a esta codificação, o intercepto estimado para cada atributo representa seu impacto médio na utilidade. Devido ao fato que os níveis dos atributos estão em uma escala comum, é possível calcular a utilidade média nessa escala, sendo possível medir (de forma comparável) o impacto médio de cada atributo nessa escala.

A seguir, foram representados graficamente os parâmetros estimados para ambas as respostas (coeficientes best vs worst) para analisar se eles diferem apenas por um escalar multiplicativo, igual à inclinação da reta para os pares de valores dos coeficientes, ou não. Em uma terceira etapa, ambas as respostas foram combinadas, admitindo utilidade sistemática igual, mas variabilidade dos erros diferentes, e modelos MNL foram estimados para o conjunto de dados combinado (best/worst). A suposição de igualdade de parâmetros realizada foi verificada com o teste da razão de verossimilhança (Ben-Akiva e Lerman 1985) (Equação 4):

$$
\lambda_{A}=-2 *\left[L_{B / W}-\left(L_{B}+L_{W}\right)\right](4)
$$

onde $L_{B / W}$ é o valor de máxima verossimilhança correspondente ao conjunto de dados combinado, $L_{B}$ é o correspondente aos dados best e $L_{w}$ aos worst. Este teste estatístico é assintoticamente distribuído qui-quadrado com $(\mathrm{K}+1)$ graus de liberdade, sendo $\mathrm{K}$ o número de parâmetros em cada conjunto de dados. O resultado indica se a hipótese de igualdade de parâmetros é verificada ou não. Quando a hipótese não pode ser rejeitada, o procedimento foi repetido para aqueles parâmetros que podiam ser considerados iguais (aqueles mais próximos da linha reta).

Finalmente, foi analisado o efeito global de cada atributo derivado dos modelos de escolha discreta. O peso do atributo $m$ pode ser estimado como (Equação 5):

$$
\text { Peso } o_{m}=\sum_{j} \frac{\exp \left(A S C_{m}\right)}{\exp \left(A S C_{j}\right)}
$$

onde $A S C_{m}$ é a constante específica do atributo (intercepto), e a soma é sobre todos os atributos presentes no desenho. Este processo de avaliação e escolha difere do utilizado em experimentos de escolha tradicionais. Neste caso, são os atributos que são os objetos de escolha em cada perfil. Portanto, cada um tem seu próprio intercepto e os pesos normalizados estimados (Equação 5) representam a probabilidade de escolha de cada atributo dentre todos os perfis considerados. As magnitudes desses pesos refletem o efeito global de cada atributo para todos os níveis utilizados na pesquisa (Louviere e Swait, 1997).

\section{COLETA DE DADOS E DESENHO DO QUESTIONÁRIO}

\subsection{Amostra e entrevista}

Entrevistas pessoais foram realizadas entre Julho e Agosto de 2014, com 390 indivíduos de 15 bairros de Porto Alegre. A amostra foi selecionada através de um método de amostragem em dois estágios: setores censitários e domicílios. Em um primeiro estágio, todos os setores censitários foram estratificados através de três variáveis: índice de motorização, densidade de comércios e serviços e inclinação média do terreno. Estas variáveis de estratificação foram escolhidas buscando captar variações na frequência de viagens a pé relacionadas a estas dimensões. Um estudo anterior realizado em Porto Alegre (Larranaga et al, 2014b) mostra que a realização de deslocamentos a pé é influenciada por estas características. Analisando os histogramas das variáveis de estratificação, para todos os setores da cidade, foram determinados os seguintes pontos de corte: (i) Índice de motorização (IM- $\mathrm{n}^{\circ}$ autom./dom): alto $(\geq 0,8)$, médio $(0,4 \leq \mathrm{IM}<0,8)$ e baixo $(<0,4)$; (ii) Declividade média: alta $(\geq 7 \%)$, baixa $(<7 \%)$ e (iii) Densidade de comércios e serviços (DC-n ${ }^{\circ}$ establec. $\left./ \mathrm{km}^{2}\right)$ : alto $(\geq 3000)$, médio-alto ( $1500 \geq$ DC $>3000)$, médio-baixo $(500 \geq \mathrm{DC}>1500)$, baixo $(<500)$. Os setores censitários foram selecionados de forma balanceada entre os estratos.

Em um segundo estágio, em cada setor censitário amostrado foram selecionados 30 domicílios através de amostragem sistemática, obtendo 26 indivíduos entrevistados em cada setor (um por domicilio). Um total de 390 domicílios dentre os 450 originalmente selecionados responderam à pesquisa, obtendo-se uma taxa de resposta de $87 \%$.

O questionário foi estruturado em duas seções: (1) características socioeconômicas da família (por exemplo, renda familiar, número de automóveis) e dos indivíduos 
Tabela 1. Estatística descritiva da amostra

\begin{tabular}{|c|c|c|}
\hline Variável & Média & Desviação Std \\
\hline Idade (anos) & 43.00 & 16.63 \\
\hline Gênero (1: masculino, 0: outro) & 0.47 & 0.50 \\
\hline Grau de Instrução Médiol (1:1ำ grau ou 2º grau com/incom, 0:outro) & 0.57 & 0.49 \\
\hline Grau de Instrução Alto (1:Superior ou Pós-graduação com/incom, 0: outro) & 0.41 & 0.49 \\
\hline Disponibilidade de automóvel $(1,0)$ & 0.50 & 0.50 \\
\hline Local de trabalho (1:fora; 0 :casa) & 0.62 & 0.48 \\
\hline Número de automóveis/domicílio (No.) & 0.81 & 0.60 \\
\hline Renda Média (1: 3300 até $\mathrm{R} \$ 6500,0$ :outro) & 0.25 & 0.43 \\
\hline Renda Alta (1:>R\$ \$ 6500, 0:outro) & 0.42 & 0.49 \\
\hline Médio № viagens a pé por semana (1: 5 até 10,0 :outro) & 0.32 & 0.46 \\
\hline Alto № viagens a pé por semana $(1:>10,0:$ outro $)$ & 0.31 & 0.46 \\
\hline
\end{tabular}

(por exemplo, idade, gênero); (2) experimento best-worst. $\mathrm{Na}$ segunda parte, um conjunto de perfis de escolha foi apresentado aos entrevistados, para que escolhessem o melhor (best) e o pior (worst) atributo, com base nos níveis considerados dentro de cada perfil de escolha (Figura 1, seção 3.2 apresenta exemplo de perfil de escolha). A Tabela 1 apresenta as características da amostra.

\subsection{Projeto experimental}

O experimento best-worst foi projetado com oito atributos, separados em dois jogos com um atributo comum, para diminuir o estresse cognitivo dos entrevistados. O primeiro jogo incluiu quatro atributos, sendo um atributo comum a ambos os jogos e três atributos associados à segurança e qualidade estética. O segundo jogo continha cinco atributos, o comum e quatro relacionados à conectividade das vias e qualidade das calçadas. Os atributos foram selecionados com base em estudos anteriores realizados em Porto Alegre (Larranaga et al 2014a, 2014b, 2014c), e especificados com 2 ou 3 níveis (ver Tabela 2). Os valores (1), (0) e (-1) apresentados na última coluna representam a codificação utilizada para cada nível (effects code).

$\mathrm{O}$ projeto experimental foi estruturado usando um desenho eficiente (Rose e Bliemer, 2009) e implementado em NGene, (Choice Metrics, 2013). O desenho eficiente foi utilizado para gerar estimativas de parâmetros com erros padrão tão pequenos quanto possível. Os erros padrão podem ser determinados através da matriz de variância-covariância (AVC), baseados na experiência subjacente e algumas informações prévias sobre as estimativas dos parâmetros. A medida de eficiência utilizada foi o D-erro, que é o determinante da matriz de AVC, para apenas um indivíduo. O objetivo é minimizar este erro de eficiência e obter um design D-ótimo (com o menor D-erro) (Rose e Bliemer, 2009).

Como não há informação prévia disponível sobre os parâmetros, foi adotado um procedimento, usual nestas situações, que envolve as seguintes etapas:

i. Projeto inicial - As estimativas dos parâmetros iniciais foram adotadas a partir de um estudo realizado em Porto Alegre (Larranaga et al, 2014b). No caso de parâmetros sobre os quais não haviam quaisquer referências (Risco acidentes, $N^{o}$ policiais, Atratividade, Largura calçada e Pavimento calçada), foi considerado apenas o sinal do parâmetro. A partir destes valores foi gerado o primeiro projeto D-eficiente.

ii. Pesquisa piloto - uma pesquisa piloto com 30 indivíduos foi realizada utilizando o projeto inicial; iii. Estimação de parâmetros - modelos MNL foram desenvolvidos para atualizar os valores iniciais dos parâmetros utilizados para criar o projeto inicial;

iv. Projeto final - com o objetivo de considerar a incerteza em relação aos parâmetros iniciais, os novos valores dos parâmetros foram utilizados para gerar um desenho eficiente Bayesiano, o qual considera que os parâmetros são aleatórios ao invés de fixos. Para o cálculo da eficiência, foi usada uma sequência de Halton. Na sequência Halton, as estimativas dos parâmetros para cada entrevistado simulado é mantida constante ao longo de várias iterações de geração de desenho, precisando de menos iterações que outros métodos. O D-erro Bayesiano (medida de eficiência utilizada para desenhos bayesianos) foi de 0,25 para o primeiro jogo e 0,18 para o segundo, valores aceitáveis para este tipo de desenhos (Choice Metrics, 2013).

O desenho final foi gerado com 18 perfis de escolha (situações de escolha) para cada jogo, separadas em três blocos (6 situações de escolha por bloco para cada jogo), de modo que cada entrevistado repetiu a tarefa de escolha das melhores e piores características para 12 situações ( 6 do jogo 1 e 6 do jogo 2). Uma pesquisa piloto foi realizada para testar o projeto e identificar problemas com o processo de pesquisa. Permitiu, por exemplo, reformular a descrição do atributo relacionado com segurança, trocando a descrição anterior de "Segurança do bairro (Seguro ou Inseguro)" por "Número de policiais cada 1.000 habitantes ( $N^{o}$ policiais $)$ " na pesquisa final. Atratividade e Conectividade foram apresentados através de imagem e texto para descrever o nível do atributo. Nove imagens para Atratividade foram criadas, a fim de representar todas as combinações entre $N^{o}$ comércios e Atratividade dentro de uma mesma situação de escolha. A Figura 1 mostra um exemplo de perfil de escolha $\mathrm{B} / \mathrm{W}$ do questionário final.

\section{RESULTADOS E DISCUSSÃO}

Os resultados dos modelos MNL estimados para cada conjunto de dados, respostas best e worst, são apresentados na Tabela 3 e graficamente na Figura 2. Na Tabela 3 são também apresentados os resultados para os modelos MNL estimados para o conjunto agrupado de dados, respostas best e worst em conjunto. 
Tabela 2. Descrição de atributos e níveis

\begin{tabular}{|c|c|c|c|}
\hline Atributo & Abreviação & $\mathbf{N}^{\circ}$ níveis & Descrição do nível \\
\hline $\begin{array}{l}\text { Número de comércios e serviços próximos da re- } \\
\text { sidência (padaria, mercado, farmácia, bares, etc) }\end{array}$ & $N^{\circ}$. Comércios & 3 & $\begin{array}{c}\text { Muitos (1), poucos }(0) \text { ou nenhum }(-1) \\
\text { comércio ou serviço próximo da residên- } \\
\text { cia }\end{array}$ \\
\hline \multicolumn{4}{|c|}{ Jogo 1} \\
\hline Risco de acidentes de tráfego & Riscos acidentes & 10 & Alto (1) ou baixo (-1) risco \\
\hline Número de policiais por 1,000 habitantes & $N^{\circ}$. Policiais & 15 & $\begin{array}{l}4 \text { policiais/1,000 habitantes (1) ou } 2 \text { (situ- } \\
\text { ação atual de Porto Alegre) }(-1)\end{array}$ \\
\hline Atratividade do bairro & Atratividade & 15 & $\begin{array}{l}\text { Muito atrativo (1), mais ou menos atra- } \\
\text { tivo (0) ou não atrativo }(-1)\end{array}$ \\
\hline \multicolumn{4}{|c|}{ Jogo 2} \\
\hline Declividade & Declividade & 28 & Terreno inclinado (1) ou plano (-1) \\
\hline $\begin{array}{c}\text { Largura útil da calçada (largura e presença de } \\
\text { obstáculos) }\end{array}$ & Largura calçada & 20 & $\begin{array}{l}\text { Calçada ampla/sem obstáculos (1), cal- } \\
\text { çada com alguns obstáculos (0) ou es- } \\
\text { treita/muitos obstáculos (-1) }\end{array}$ \\
\hline Condições do pavimento da calçada & Pavimento calçada & 26 & Boa (1) ou más condições (-1) \\
\hline Conectividade viária & Conectividade & 15 & $\begin{array}{c}\text { Muitos (1) ou poucos (-1) caminhos al- } \\
\text { ternativos para ir de uma origem a um } \\
\text { destino }\end{array}$ \\
\hline
\end{tabular}

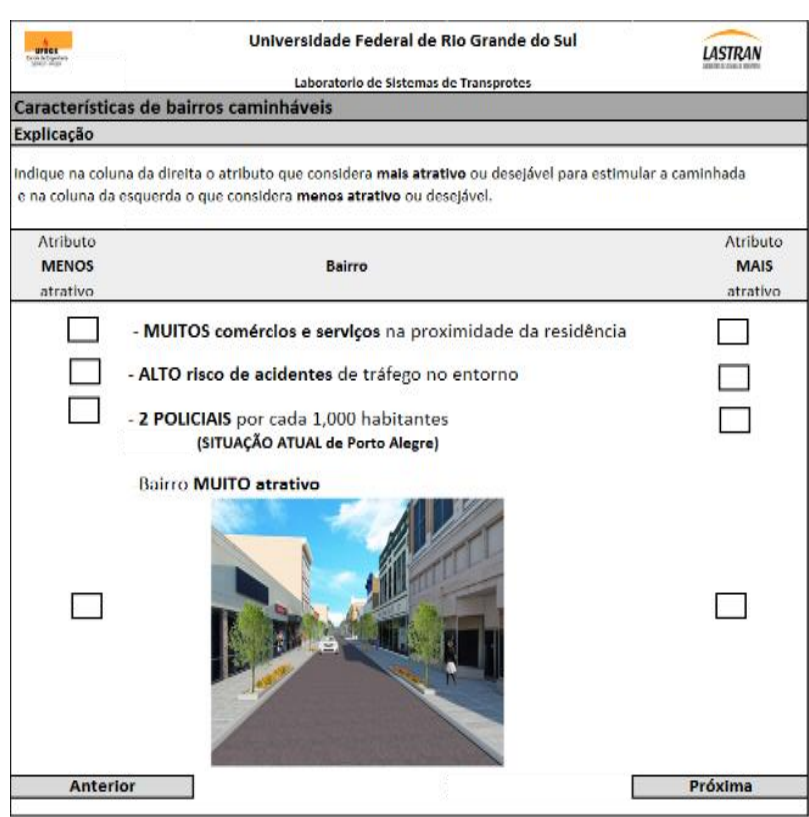

Figura 1. Exemplo de perfil de escolha B/W para o jogo 1

A linha rotulada como "Efeito de..." contêm o valor da constante específica ASC (intercepto) para cada modelo, o que representa o efeito global de cada atributo, conforme estimado pelo MNL. A linha rotulada "Nível do atributo" contêm o valor do coeficiente estimado para cada atributo. Modelos com fator de escala diferente para cada jogo foram estimados, mas estes não foram significativamente diferentes (Fator de escala jogo1/jogo 2=1). O sinal dos coeficientes estimados para os atributos estão de acordo com os pressupostos. Maior número de comércios no entorno, baixo risco de acidentes de tráfego, maior número de policiais, bairro mais atrativo, boa conectividade entre origem e destino, terreno plano e calçadas largas e em boas condições estimulam o deslocamento a pé.

A Figura 2 apresenta o gráfico de confronto dos parâmetros do modelo Best e Worst. O gráfico sugere que os coeficientes de ambos os modelos podem diferir apenas por um escalar multiplicativo, apresentando uma relação linear entre eles (linha reta). Assim, sugere que os dados best e worst poderiam ser combinados considerando coeficientes iguais em ambos os modelos.
Portanto, foi estimado um modelo MNL para o conjunto de dados combinados, respostas best e worst em conjunto (Tabela 3 ) e verificado se os coeficientes efetivamente são iguais nas respostas best e worst, mas com fatores de escala diferente. Para isto, foi utilizado o teste de razão de verossimilhança (Equação 4) e comparados os três modelos. O valor do logaritmo da função de verossimilhança do modelo conjunto foi $-7115.26\left(\mathrm{~L}_{\mathrm{B} / \mathrm{w}}\right)$, e fator de escala relativo corresponde a 1,08 (significativamente diferente de 1). A estatística qui-quadrado foi 223,90, com 14 graus de liberdade, e o valor crítico da distribuição qui-quadrado correspondente para o nível de confiança de $95 \%$ é 23,68. Assim, a hipótese de igualdade parâmetros entre os modelos Best e Worst é rotundamente rejeitada.

A Figura 2 mostra que alguns coeficientes estão mais afastados da linha reta do que outros, indicando que poderiam ser diferentes entre os conjuntos de dados best e worst. Os atributos Largura calçada, Pavimento calçada, $\mathrm{N}^{o} \mathrm{co}$ mércios e $N^{o}$ policiais parecem ser os mais diferentes entre os modelos (mais afastados da linha reta). As constantes específicas garantem que as proporções de mercado são replicadas pelo modelo MNL (Ben Akiva e Lerman, 1985). Por isso, se assume igualdade dos coeficientes, mas se permite que as proporções agregadas das alternativas difiram (não se exige igualdade de interceptos).

A hipótese de igualdade de todos os coeficientes foi rejeitada, por isso foi repetido o procedimento para aqueles coeficientes que poderiam ser considerados iguais. O processo foi iniciado considerando Largura calçada como específico a cada conjunto de dados. Como a hipótese de igualdade de coeficientes neste teste foi rejeitada, foi estimado um novo modelo Best/Worst incluindo os atributos Largura de calçada e Pavimento calçada como específicos a cada conjunto. Sendo as hipóteses rejeitadas procedia-se a estimativa de um novo modelo com a agregação de um novo atributo. A hipótese de igualdade de parâmetros dos atributos foi rejeitada em todos os casos. Este resultado implica que o julgamento e escolha dos melhores e piores atributos têm modelos subjacentes diferentes, sugerindo que cada escolha é realizada por um processo cognitivo diferente. 
LARRAÑAGA, A.M.; CYBIS, H.B.B.; ARELLANA, J.; RIZZI, L.I; STRAMBI.O.

Tabela 3. Resultados dos modelos MNL: Best, Worst, Best/Worst

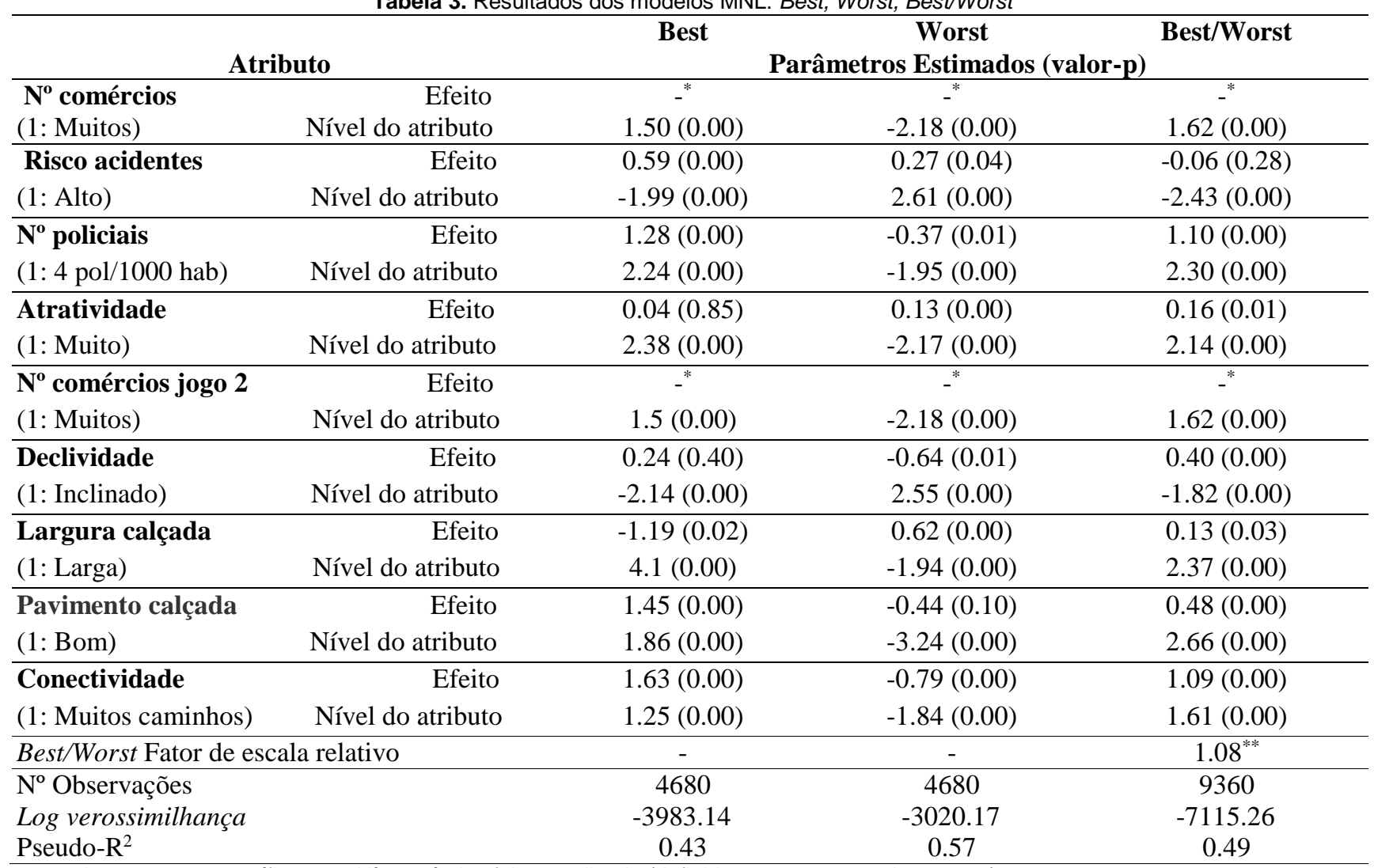

nota1: A constante específica para № comércios foi normalizada (definida em zero para o jogo 1 e 2)

nota2: Fator de escala significativamente diferente de 1.

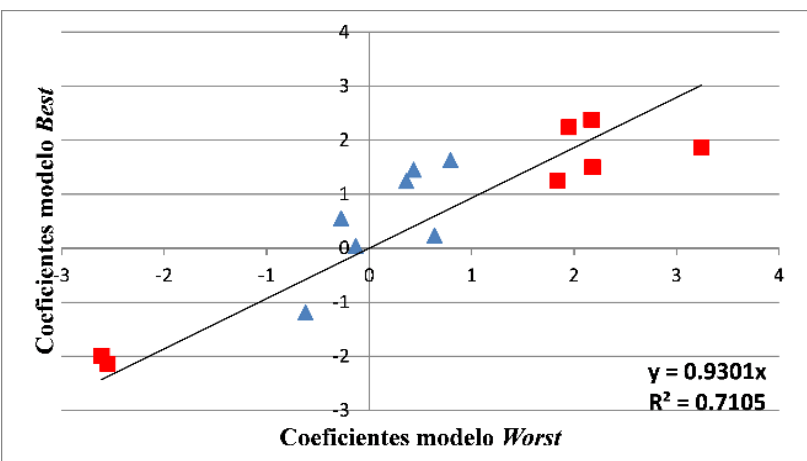

Figura 2. Parâmetros do MNL- Best versus Worst

Os entrevistados visualizam as características do ambiente construído do bairro para estimular as viagens utilitárias pé de forma diferente quando escolhem as características mais atrativas e quando escolhem as menos atrativas.

A análise do efeito global de cada atributo derivado dos modelos MNL foi realizada através da estimação do impacto normalizado utilizando a Equação 5. Figura 3 ilustra a importância das características do ambiente construído resultantes de estimação dos modelos Best e Worst. Quanto maior a pontuação, mais importante é o atributo.

A importância obtida a partir das respostas best está relacionadas com as características mais desejadas. Os entrevistados avaliam os atributos de uma forma positiva, identificando aqueles mais atraentes para estimular a caminhada. As quatro características mais importantes são (Figura 3): Conectividade, Pavimento calçada, $N^{o}$ policiais e Risco acidentes. A hierarquização dos atributos obtida das respostas best é igual à obtida em um estudo anterior para
Porto Alegre (Larranaga et al, 2014b) aplicando uma abordagem completamente diferente. A hierarquização obtida nesse estudo, determinada através do cálculo de elasticidades, foi: conectividade, segurança (risco de acidentes e segurança pública medida como o efeito indireto sobre uma variável latente), declividade e número de comércios. Atributos relativos a calçadas e atratividade não foram considerados no estudo.

Por outro lado, as importâncias obtidas a partir das respostas worst estão relacionadas com as características menos desejáveis. Os entrevistados avaliam os atributos de uma forma negativa, identificando aqueles que são menos atraentes. As quatro características mais importantes identificadas são: Conectividade, Declividade, Pavimento calçada e $N^{o}$ policiais. Declividade teve um efeito maior do que na análise das respostas best. Os outros três foram importantes em ambas as análises. Nos atributos extremos, Conectividade e Largura calçada os resultados de ambas respostas coincidem.

Esperávamos um impacto maior de $N^{o}$ policiais. A pesquisa foi realizada após a Copa do Mundo de 2014, na qual Porto Alegre foi cidade sede e a segurança da cidade reforçada. Embora o entrevistador reforçasse que o atributo se referia à situação habitual de Porto Alegre, não para o período da Copa, isto pode ter interferido na mensuração do atributo. Os resultados mostram que o julgamento e escolha dos atributos do bairro mais e menos atraentes para estimular a caminhada são medidos por um processo cognitivo diferente e o efeito (importância) de cada um também é diferente. 


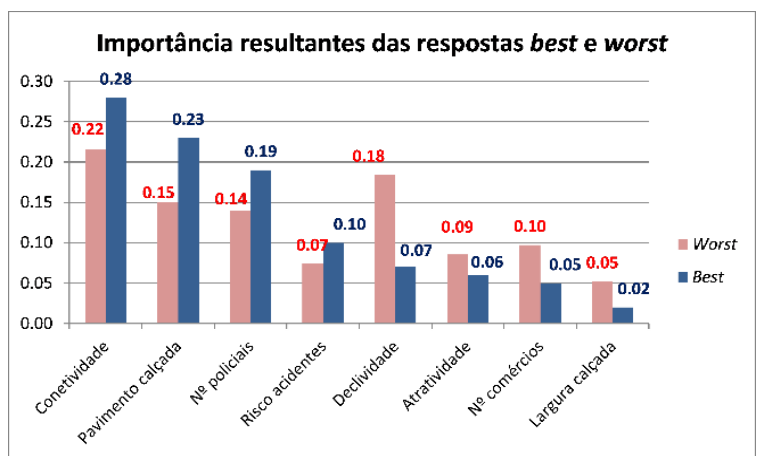

Figura 3. Importância das características resultantes das respostas best e worst

\section{CONCLUSÕES}

Este estudo determina a importância relativa de características do ambiente construído para promover bairros caminháveis usando Best-Worst Scaling, no contexto da cidade de Porto Alegre. A principal contribuição do método adotado é que permite identificar separadamente o impacto dos atributos e o impacto dos níveis utilizados para medir os atributos no experimento, algo que não possível com qualquer forma de análise conjunta tradicional.

Coletar respostas best e worst contribui com maior informação sobre a avaliação subjetiva de características do ambiente construído pelos residentes de Porto Alegre, a fim de calcular a importância de cada atributo. No entanto, os resultados mostram que o julgamento e escolha dos melhores e piores atributos são realizados por processos cognitivos diferentes. Os entrevistados visualizam as características do ambiente construído do bairro para estimular as viagens utilitárias pé de forma diferente quando escolhem as características mais atrativas do que quando escolhem as menos atrativas. A importância obtida a partir de cada tipo de resposta também difere. Importância dos atributos é mais similar nos atributos extremos do que entre os itens de importância média.

As características mais importantes para estimular a caminhada obtidas a partir de respostas best são: Conectividade, Pavimento Calçada, $N^{o}$ policiais e Risco acidentes. Por outro lado, as mais importantes obtidas a partir das respostas worst são: Conectividade, Declividade, Pavimento calçada e $N^{o}$ policiais.

A análise sugere que a utilização de respostas best ou worst depende do objetivo do estudo. Respostas best poderiam ser usadas na análise do impacto de mudanças no ambiente construído para aumentar a frequência de viagens a pé. O desenvolvimento de estratégias de planejamento urbano que visem aumentar o número de deslocamentos a pé estarão mais preocupados em identificar os resultados best (aqueles que são mais desejáveis para caminhar) do que os resultados worst. Respostas worst poderiam ser usadas para a identificação de barreiras ou impedimentos existentes para o pedestre. Os resultados obtidos a partir das respostas worst podem oferecer maior valor prático no desenvolvimento de estratégias urbanas que visam eliminar barreiras à caminhabilidade.

\section{REFERÊNCIAS}

Adamsen, J.M. e Whitty, J.A.(2013) Best-Worst scaling reflections on presentation, analysis, and lessons learnt from case 3. Market \& Social Research, v.21, n.1. Disponível em: http://www.amsrs.com.au/documents/item/1102 . Acesso em: 03/02/2015.

City of Fort Collins (2002) Pedestrian level-of-service. Fort Collins, CO. Disponível em:

<http://www.fcgov.com/transportationplanning/pedplan.php> Acesso em: 12/12/2014.

Auger, P.; Devinney, T.M. e Louviere, J. (2007) To What Extent Do Consumer Ethical Beliefs Differ Across Countries? A CrossCountry Investigation Using Best-Worst Scaling Methodology. Journal of Business Ethics v.3, n.70,p.299-326. DOI: 10.1007/s10551-006-9287-y

Baran, P.; Rodríguez, D. e Khattak, A.(2008) Space Syntax and Walking in a New Urbanist and Suburban Neighborhoods, Journal of Urban Design, v.1, n.13, p.5-28. DOI: 10.1080/13574800701803498

Ben-Akiva, M. e Lerman, S.R. (1985) Discrete Choice Analysis: Theory and Application to Travel Demand. Cambridge, MA: MIT Press.

Bradshaw, C. (1993) Creating and using a rating system for neighborhood walkability: Towards an agenda for "local heroes. ' Paper presented at the 14th International Pedestrian Conference, Boulder, CO. Disponível em:

http://hearthhealth.wordpress.com/about/previously-publishedworks/feet-first-early/creaing-and-using-a-rating-system-forneighbourhood-walkability-towards-an-agenda-for-local-heroes1993/ . Acesso em: 02/01/2015

Burge, P.; Potoglou,D. e Flynn, T. Brazier,J.E. e Netten, A. (2011) Best worst scaling: Consistency of preferences with discrete choice experiments and stability over time. International Choice Modelling Conference, Leeds. Disponível em : http://www.icmconference.org.uk/index.php/icmc/ICMC2011/pa per/view/327. Acesso em: 12/01/2015

Burke, P.F; Louviere, J. Wei, E.; MacAulay, G.; Quail, K. e Carson,R. (2013) Overcoming Challenges and Improvements in Best-Worst Elicitation: Determining What Matters to Japanese Wheat Millers. Open Conference Systems Disponível em: http//www.opus.lib.uts.edu.au Acesso em 03/02/2015

Cao, X.; Handy, S. e Mokhtarian, P. (2006) The Influences of the Built Environment and Residential Self-Selection on Pedestrian Behavior, Tx. Transportation, v.1, n.33, p.1-20. DOI: 10.1007/s11116-005-7027-2

Cervero, R. e Duncan, M. (2003) Walking, bicycling, and urban landscapes: evidence from the San Francisco Bay Area. Am J Public Health, v.9, n.93, p.1478-1483. DOI: 10.2105/AJPH.93.9.1478

Choice Metrics (2013) Ngene 1.1 User Manual and Reference Guide. Choice Metrics. Disponível em: http://www.choicemetrics.com/download.html. Acesso em: 5/11/2014.

Cohen, E. (2009) Applying Best-Worst Scaling to Wine Marketing. International Journal of Wine Business Research, v. 1, n.21, p. 8-23. DOI: $10.1108 / 17511060910948008$

Coltman, T. R., Devinney, T. M. e Keating, B. W.(2011) BestWorst Scaling Approach to Predict Customer Choice for 3PL 
Services. Journal of Business Logistics, v.2, n.32, p.139-152. DOI: $10.1111 / \mathrm{j} .2158-1592.2011 .01012 . x$

Dannenberg, A. (2004) Assessing the walkability of the workplace: A new audit. Presented at the 4th National Congress of Pedestrian Advocates, America Walks. DOI: 10.4278/08901171-20.1.39

Ewing, R. e Cervero, R. (2010) Travel and the Built Environment -- A Meta-Analysis. Journal of the American Planning Association v.76, p.265-294. DOI: $10.1080 / 01944361003766766$

Flynn, T.N. (2010) Using conjoint analysis and choice experiments to estimate quality adjusted life year values: issues to consider. Pharmacoeconomics v.28, p.711-722. DOI: 10.2165/11535660-000000000-00000

Flynn, T.; Louviere, J.; Peters, T. e Coast, J.(2007) Best-worst scaling: What it can do for health care research and how to do it. Journal of Health Economics, v.26, p.171-189. DOI: 10.1016/j.jhealeco.2006.04.002

Frank, L.D. e Engelke, P.O.(2001) The Built Environment and Human Activity Patterns: Exploring the Impacts of Urban Form on Public Health. Journal of Planning Literature, v.16, p.202218. DOI: $10.1177 / 08854120122093339$

Jaeger,S; Jørgensen,R. Aaslyng,A.;Bredie,A.e Wender,L.P.(2008)Best-worst scaling: An introduction and initial comparison with monadic rating for preference elicitation with food products. Food Quality and Preference v.6, n.19, p.579-588. DOI: 10.1016/j.foodqual.2008.03.002

Krambeck, H.V. (2006) The Global Walkability Index. Department of Urban Planning and Department of Civil and Environmental Engineering, Massachusetts Institute of Technology. Disponível em: : http://hdl.handle.net/1721.1/34409. Acesso em: 01/05/2014.

Lancsar, E.; Louviere,J. e Flynn, T. (2007) Several methods to investigate relative attribute impact in stated preference experiments. Social Science \& Medicine, v.8, n.64, p.1738-53. DOI: $10.1016 / j$.socscimed.2006.12.007

Larranaga, A. M. L.; H.B. Cybis e O. Strambi (2014a) The Influence of Urban Form Perceived Characteristics and Attitudinal Values on Walking: A Case Study of Porto Alegre, Brazil. TRB 93rd Annual Meeting Compendium of Papers, p.14.

Larranaga, A. M. L.; L. I. Rizzi; J. Arellana; O. Strambi e H. Cybis (2014b) The Influence of built environment and travel attitudes on walking: a case study of Porto Alegre, Brazil. International Journal of Sustainable Transportation. DOI: 10.1080/15568318.2014.933986

Larranaga, A.M., H.B. Cybis e O. Strambi (2014c) Determinação da importância relativa dos atributos do bairro que estimulam as viagens a pé. In: XXVIII ANPET Congresso de Pesquisa e Ensino em Transportes. Curitiba.

Lee, J. A., Soutar, G.N., e Louviere, J. (2007) Measuring Values

Using Best-Worst Scaling: The LOV Example. Psychology \& Marketing, 24(12), 1043-1058. DOI: 10.1002/mar.20197

Lee, C. e Moudon, A.V. (2006) Correlates of Walking for Transportation or Recreation Purposes, Journal of Physical Activity and Health, v.1, n.3, p.77-98.
Louviere, J. e Swait, J.D.(1997) Separating weights and scale values in conjoint tasks using choices of best and worst attribute levels. Technical Report, Centre for the Study of Choice, University of Technology Sydney. DOI: 10.13140/RG.2.1.2526.7924

Louviere, J., e Woodworth, G.(1990) Best-worst scaling: A model for largest difference judgments. Report, Faculty of Business, University of Alberta.

Marley, A.A. e Louviere, J.(2005) Some probabilistic models of best, worst, and best-worst choices. Journal of Mathematical Psychology v.49,p.464-480. DOI: 10.1016/j.jmp.2005.05.003

Rose, J.M.e Bliemer,M.C.(2009) Constructing Efficient Stated Choice Experimental Designs. Transport Reviews, v.5, n.29, p.587-617. DOI: 10.1080/01441640902827623

Swait, J.D.e Louviere, J.(1993) The Role of the Scale Parameter in The Estimation and Comparison of Multinomial Logit Models, Journal of Marketing Research, v.30, p.305-314. DOI: $10.2307 / 3172883$

Walkscore (2010) Find a Walkable Place to Live. Disponível em: http://www.walkscore.com/ Acesso: 1/10/2014 